\title{
Effect of Patient Sex on White Matter Alterations in Unilateral Medial Temporal Lobe Epilepsy with Hippocampal Sclerosis Assessed by Diffusion Tensor Imaging
}

\author{
K.K. Oguz, I. Tezer, E. Sanverdi, A.C. Has, B. Bilginer, A. Dolgun, and S. Saygi
}

\begin{abstract}
BACKGROUND AND PURPOSE: Studies shows ictal behavior and symptoms are affected by patient sex in temporal lobe epilepsy. The purpose of our study was to determine whether alterations in the WM as assessed by DTI display different patterns in male and female patients with unilateral HS.
\end{abstract}

\begin{abstract}
MATERIALS AND METHODS: Patients with unilateral HS were categorized as women with right HS ( $n=12$ ), men with right HS ( $n=10$ ), women with left HS $(n=12)$, and men with left HS $(n=10)$. DTI of the brain along 64 noncollinear directions was obtained from 44 patients and 37 sex-matched control participants. We used TBSS to analyze whole-brain WM. Regions with significant changes of FA and $M D$, and their mean FA, MD, total number of significant voxels, and asymmetry indices were determined for each group.

RESULTS: All groups showed bilateral and extensive reductions of FA and elevated MD in the WM, more prominent ipsilateral to the affected hippocampus. The total number of voxels with decreased FA in patients compared with that of control participants was higher in women with right $\mathrm{HS}(24,727$ vs 5,459$)$ and in men with left $\mathrm{HS}(27,332$ vs 14,013$)$ than in their counterparts. Changes in MD associated with right HS were more extensive in both men and women (right vs left HS, women: 16,926 vs 5,458; men: 5,389 vs 4,764) than in those with left HS. In patients with right HS, the ipsilateral cingulum, uncinate fasciculus, internal and external capsules, and right acoustic radiation were involved extensively in women.
\end{abstract}

CONCLUSIONS: Women and men showed different patterns in extent of WM alterations associated with HS.

ABBREVIATIONS: $\mathrm{Al}=$ asymmetry index; EEG, electroencephalography; $\mathrm{FA}=$ fractional anisotropy; $\mathrm{HS}=$ hippocampal sclerosis; $\mathrm{MD}=$ mean diffusivity; TBSS = tract-based spatial statistics; TLE $=$ temporal lobe epilepsy

$\mathbf{T}$ LE is the most frequent type of partial epilepsy, and unilateral HS is diagnosed reliably with MR imaging in a majority of patients. The pathologic findings mainly consist of neuronal loss and gliosis in the hippocampal formation, amygdala, parahippocampus, and entorhinal cortex. ${ }^{1,2}$ Recent neuroimaging studies on unilateral TLE with and without HS have further demonstrated extensive WM abnormalities with some variability. ${ }^{3,4}$ Although the cause and clinical implications are yet to be specified, it has been suggested that the WM changes may represent a secondary effect of ongoing seizure caused by axonal trans-

Received June 6, 2012; accepted after revision July 23.

From Bilkent University, National Magnetic Resonance Research Center (K.K.O., A.C.H.), Bilkent, Ankara, Turkey; and Faculty of Medicine, Departments of Radiology (K.K.O., E.S.), Neurology (I.T., S.S.), Neurosurgery (B.B.), and Biostatistics (A.D.), Hacettepe University, Sihhiye, Ankara, Turkey.

Please address correspondence to Kader K. Oguz, Bilkent University, National Magnetic Resonance Research Centre (UMRAM), Cyberplaza, CBlock. Level 2, BilkentAnkara, Turkey; e-mail: karlioguz@yahoo.com

http://dx.doi.org/10.3174/ajnr.A3328 mission $^{5}$ and reflect abnormal networks associated with epileptogenesis. ${ }^{6}$

Because diffusion perpendicular to the main fiber direction is more hindered by axon membranes and myelin than diffusion parallel to the main axis, and therefore anisotropic in the WM, DTI provides unique information about microstructural tissue properties such as axonal organization and myelin attenuation that cannot be obtained from conventional T1- or T2-weighted images. The most commonly used DTI indices are FA and MD, reflecting the degree of WM integrity and the presence of cell membranes or myelin sheaths (ie, diffusion barriers). ${ }^{7}$ Both mesial and neocortical unilateral TLE generally have shown widespread temporal and extratemporal WM abnormalities on DTI studies. $^{5,8,9}$

Previous animal models and human imaging studies, including voxel-based morphometry and DTI, have revealed inherent morphologic and functional variations between men and women. Some of these variations include thicker cortices in the right inferior parietal and posterior temporal regions in women, which are present from childhood and are maintained throughout life $^{10}$; 


\begin{tabular}{|c|c|c|c|c|c|c|}
\hline Group & $\begin{array}{l}\text { No of } \\
\text { Patients } \\
(n)\end{array}$ & $\begin{array}{c}\text { Age Range; } \\
\text { Mean Age } \pm \text { SD } \\
\text { (y) }\end{array}$ & $\begin{array}{c}\text { Duration of } \\
\text { Epilepsy; Mean } \pm \text { SD } \\
(y)\end{array}$ & $\begin{array}{l}\text { History of } \\
\text { Risk Factors } \\
(n)\end{array}$ & $\begin{array}{c}\text { Unilateral } \\
\text { Ipsilateral } \\
\text { Interictal EEG } \\
\text { Abnormality } \\
\text { (n) }\end{array}$ & $\begin{array}{c}\text { Bilateral } \\
\text { Interictal EEG } \\
\text { Abnormality } \\
\text { (n) } \\
\end{array}$ \\
\hline Women with right HS & 12 & $\begin{array}{c}19-47 ; 30.58 \pm 9.78 \\
{ }^{\mathrm{a}} P=.89 ;{ }^{\mathrm{b}} P=.94 \\
{ }^{\mathrm{d}} P=.73\end{array}$ & $\begin{array}{l}1-38 ; 20.41 \pm 10.69 \\
\mathrm{~b} P=.98 ;{ }^{d} P=.47\end{array}$ & $\begin{array}{l}\text { FS: 9; FH: 4; trauma: 1; } \\
\text { meningitis: } 1 \text {; none: } 2\end{array}$ & 12 & None \\
\hline Men with right HS & 10 & $\begin{array}{c}17-42 ; 30.9 \pm 8.72 \\
{ }^{\mathrm{a}} P=.93 ;{ }^{\mathrm{b}} P=.94 \\
\text { e } P=.55\end{array}$ & $\begin{array}{l}6-41 ; 20.55 \pm 12.12 \\
{ }^{\mathrm{b}} P=.98 ;{ }^{\mathrm{e}} P=.30\end{array}$ & $\begin{array}{c}\text { FS: } 5 \text {; FH: } 3 \text {; trauma: } 2 ; \\
\text { none: } 3\end{array}$ & 7 & 3 \\
\hline Women with left HS & 12 & $\begin{array}{c}23-37 ; 29.5 \pm 4.21 \\
{ }^{\mathrm{a}} P=.81 ;{ }^{c} P=.20 \\
{ }_{\mathrm{d}} P=.73\end{array}$ & $\begin{array}{l}16-28 ; 20.44 \pm 5.10 \\
{ }^{c} P=.13 ;{ }^{d} P=.47\end{array}$ & $\begin{array}{c}\mathrm{FS}: 7 \text {; FH: } 4 \text {; trauma: } 3 \text {; } \\
\text { none: } 4\end{array}$ & 11 & 1 \\
\hline Men with left HS & 10 & $\begin{array}{c}22-42 ; 32.7 \pm 7.02 \\
{ }^{\mathrm{a}} P=.69 ;{ }^{c} P=.20 \\
\mathrm{e} P=.55\end{array}$ & $\begin{array}{c}10-36 ; 25.60 \pm 8.42 \\
{ }^{c} P=.13 ;{ }^{~} P=.30\end{array}$ & $\begin{array}{l}\text { FS: 4; FH: } 4 \text {; trauma: } 5 \text {; } \\
\text { perinatal insult: } 2 \text {; none: } 1\end{array}$ & 10 & None \\
\hline
\end{tabular}

Note:-Lateralization of HS on MR imaging and electrophysiologic ictal findings, and unilateral or bilateral presence of interictal EEG abnormalities of the patients are summarized.

FH indicates family history, FS, febrile seizure.

a $P$ values for matched control participants.

${ }^{b} P$ values for women with right $\mathrm{HS}$ and men with right $\mathrm{HS}$.

c $P$ values for women with left $\mathrm{HS}$ and men with left HS.

${ }^{d} P$ values for women with right $\mathrm{HS}$ and women with left $\mathrm{HS}$.

${ }^{e} P$ values for men with right $\mathrm{HS}$ and men with left $\mathrm{HS}$.

different intrahemispheric or interhemispheric cerebral metabolic rates for glucose $\mathrm{e}^{11}$; and differences in WM architecture as inferred from diffusion properties of the brain tissue. ${ }^{12}$ The effect of sexual dimorphism in TLE associated with HS has also been stressed for variable seizure semiology in many studies. Ictal fear, ${ }^{13}$ vocalization, ${ }^{14}$ vegetative signs, ${ }^{15}$ and sexual auras ${ }^{16}$ occur more frequently in women than in men. Contrary to this wide range of sex-dependent symptoms, to the best of our knowledge, no study has investigated the influence of sexual dimorphism on WM changes associated with HS in the brain. Given that intrahemispheric and interhemispheric anatomic and functional connections may determine seizure spread and ictal behavior in male and female patients, we hypothesized that alterations in the WM as assessed by DTI display different patterns in male and female patients with HS.

\section{MATERIALS AND METHODS \\ Participants}

We conducted this prospective study in a tertiary care center with a dedicated epilepsy team including epileptologists, neurosurgeons, neuroradiologists, and psychologists. The local institutional review board approved the study. All participants gave written informed consent before inclusion into the study.

The patients were retrieved from a data base integrated with Hospital and Radiology Information Systems. Inclusion criteria of this study were clinically and/or electrophysiologically welldefined stereotyped temporal-type seizures, the presence of unilateral HS, and the absence of other abnormalities on structural MR imaging. Exclusion criteria were the presence of bilateral HS and the presence of any additional abnormality (dual pathologic abnormality) on structural MR imaging, and associated neuropsychiatric or systemic illness.

A total of 44 right-handed patients with HS and 37 sexmatched healthy control participants were categorized into 4 groups: women with right $\mathrm{HS}(n=12$; mean age, $30.58 \pm 9.78$ years) and control participants $(n=10$; mean age, $31.2 \pm 10.03$ years), men with right HS ( $n=10$; mean age, $30.9 \pm 8.72$ years) and control participants ( $n=9$; mean age, $30.55 \pm 7.40$ years $)$, women with left HS ( $n=12$; mean age, $29.5 \pm 4.21$ years $)$ and control participants ( $n=9$; mean age, $29 \pm 5.4$ years $)$, and men with left HS ( $n=10$; mean age, $32.7 \pm 7.02$ years $)$ and control participants $(n=9 ; 31.44 \pm 6.18$ years $)$. Groups matched for sex and lateralization of HS were similar in distributions of age (all $P>.69$ ) and duration of epilepsy (all $P>$ .1). A total of 34 patients had 1 or more risk factors for HS: a history of febrile seizure(s) $(n=25)$, traumatic injury $(n=$ $11)$, meningitis $(n=1)$, perinatal insult $(n=2)$, and a family history of epilepsy $(n=15)$.

Demographic and clinical features, lateralization of the affected hippocampus found on MR imaging, electrophysiological ictal abnormalities, and laterality of interictal EEG findings in the patients are summarized in Table 1.

None of the patients had a seizure within the last 3 days at the time of MR imaging.

Healthy control participants $(n=37)$ were volunteers who had no neuropsychiatric or systemic disease. These volunteers were recruited via local announcements in the hospital and university. All had normal findings on MR imaging.

All of the patients and control participants were right-handed, as determined by their preference on handwriting.

Two neuroradiologists, one of whom has 15 years of experience in epilepsy imaging (K.K.O.), evaluated structural brain MR imaging for the 2 major findings of HS (ie, decreased thickness and increased T2 signal intensity of the hippocampus), recorded lateralization of the HS, and reviewed images for dual pathologic findings in consensus.

Outpatient routine and long-term video-EEG monitoring records of the patients were reviewed by 2 experienced epileptologists (I.T., S.S.) in consensus. Age; the presence of risk factors for 
HS (ie, a history of febrile seizure, traumatic injury, or meningitis; and a family history of epilepsy); duration of epilepsy; and abnormal activities in the interictal and ictal EEG, when available, were recorded for each patient.

\section{Image Acquisition}

We performed all imaging studies on a 1.5T MR scanner (Magnetom, Symphony TIM system; Siemens, Erlangen, Germany) equipped with a $30-\mathrm{mT} / \mathrm{m}$ gradient system by using an 8 -channel phased array head coil. Conventional brain MR pulse sequences were axial T1-weighted spin-echo (TR, $500 \mathrm{~ms}$; TE, $20 \mathrm{~ms}$ ), axial fast fluid-attenuated inversion recovery (TR, $9000 \mathrm{~ms}$; TE, 100 $\mathrm{ms}$; TI, $2100 \mathrm{~ms}$ ), and coronal T2-weighted fast spin-echo perpendicular to the hippocampi (TR, $4000 \mathrm{~ms}$; TE, $100 \mathrm{~ms}$; section thickness, $3 \mathrm{~mm}$ ).

DTI applied a single-shot echo-planar imaging sequence and was performed in the axial plane, parallel to the anteroposterior commissures. The following parameters were used: TR, $5814 \mathrm{~ms}$; TE, $98 \mathrm{~ms}$; maximum b factor, $1000 \mathrm{~s} / \mathrm{mm}^{2}$; 64 independent directions; field of view, $23 \mathrm{~cm}$; matrix, $128 \times 128$; and number of sections, 50, with 3-mm thickness without an intersection gap covering the entire brain.

\section{Data Processing and Analyses}

Using TBSS as a part of the FMRIB Software Library 4.0 package (Oxford Centre for Functional MRI of the Brain, Oxford University, Oxford, United Kingdom; http://www.fmrib.ox.ac.uk/fsl), we performed voxelwise statistical analysis of FA and MD maps separately for each category of HS and its matched control group. Additional TBSS analysis was also carried out to document WM diffusion abnormalities in female vs male control participants. Preprocessing of the diffusion-weighted data included head motion and eddy current correction, diffusion tensor fitting (FSL DTIFit), and calculation of the FA and MD maps. The FA and MD maps were registered and aligned to the average space as input for TBSS, and the thinned mean FA skeleton was computed. Then, we performed voxelwise statistics on FA and MD by using the permutation-based inference with 500 permutations. Resulting threshold-free cluster enhancement output was corrected for multiple comparisons, and family-wise error-corrected maps were obtained with $P$ values $<.05$. We then used standard clusterbased thresholding corrected for multiple comparisons. After we calculated the total number of voxels with significantly low FA and high MD, we used Freeview (http://surfer.nmr.mgh. harvard.edu/fswiki/FreeviewGuide) to separate these clusters and number of significant voxels into each hemisphere. AI was also calculated for each patient group by use of the formula ( $\mathrm{L}-\mathrm{R} /$ $\mathrm{L}+\mathrm{R}) \times 100$, where $\mathrm{L}$ and $\mathrm{R}$ designate the number of significant voxels in the left and right hemispheres, respectively. Therefore, positive and negative results pointed out dominancy of significant voxels in the left and right hemispheric WM in the same order.

WM clusters with significant FA and MD change on the corrected threshold cluster extend voxel maps were extracted as ROIs and were registered to and overlaid onto an anatomic Montreal Neurological Institute template. We then labeled these ROIs by using the Johns Hopkins University WM tractography atlas
Table 2: Number and Al of voxels with statistically significant decreased FA and MD

\begin{tabular}{lcc}
\hline \multicolumn{1}{c}{ Group } & $\begin{array}{c}\text { No. of Voxels with } \\
\text { Significant Change } \\
\text { (FA/MD) }\end{array}$ & $\begin{array}{c}\text { Al of Voxels } \\
\text { (L-R/L+R } \times 100) \\
\text { (FA/MD) }\end{array}$ \\
\hline Women with right HS & $24,727 / 16,926$ & $-41.4 /-29.2$ \\
Men with right HS & $14,013 / 5389$ & $-19.7 /-62.2$ \\
Women with left HS & $5459 / 5458$ & $32.9 / 41.6$ \\
Men with left HS & $27,332 / 4764$ & $10.9 / 22.04$ \\
\hline
\end{tabular}

and the International Consortium for Brain Mapping DTI-81 WM atlas. The mean FA and MD of the ROIs were calculated for each participant; mean values and $\mathrm{x}-$, $\mathrm{y}^{-}$, and $\mathrm{z}$-coordinates according to the Montreal Neurological Institute atlas were also recorded.

We performed statistical analyses using SPSS for Windows, version 17.0 (SPSS, Chicago, Illinois). The Kruskal-Wallis test, the Bonferroni-adjusted Mann-Whitney $U$ test, and the $\chi^{2}$ test were used to test group differences in age and duration of epilepsy. $P$ values $<.05$ were considered significant.

\section{RESULTS}

Within the control group, the difference between men and women was subtle. There were few voxels with less FA and higher $\mathrm{MD}$ in men compared with women.

When compared with the control participants, numerous WM structures showed reduced FA and elevated MD in patients with HS. Overall, the greatest extent of change was observed in men with left HS followed by women with right HS for FA, and in women with right HS for MD. Right HS in women and left HS in men were associated with more extensive FA reductions than their counterparts. The extent of elevated MD values was 3-fold greater in women with right HS than in women with left HS and was slightly greater in men with right HS than in men with left HS. The total numbers of voxels with significantly lower FA and higher MD compared with those of the control participants and AI for each group are summarized in Table 2.

With lateralization of HS, women showed a greater extent of FA and MD changes within the right HS groups. In the patients with left HS, reduction in FA was more extensive in men, with a slightly greater extent of MD increase in women.

All 4 groups showed regions with decreased FA and increased MD in temporal and extratemporal WM. This effect was more prominent in the hemisphere ipsilateral to the HS. However, predominant FA reduction of WM ipsilateral to the seizure focus was more pronounced in women regardless of lateralization of HS. Within all groups, AI was greatest in the patients with right HS: in women for FA changes $(-41.4)$ and in men for MD changes $(-62.2)$.

The $\mathrm{x}^{-}, \mathrm{y}-$, and $\mathrm{z}$-coordinates according to the Montreal Neurological Institute atlas, the corresponding WM structures according to the Johns Hopkins University WM tractography International Consortium for Brain Mapping DTI-81 WM atlases, and the mean FA and MD values of significant clusters are given in Table 3. The cingulum, anterior temporal lobe WM, fornix, genu and body of the corpus callosum, the internal and external capsules, inferior longitudinal and inferior fronto-occipital fasciculus, thalamus, and uncinate fasciculus were shared affected 
Table 3: Summary of TBSS analysis

\begin{tabular}{|c|c|c|c|c|c|c|c|c|}
\hline \multirow[b]{2}{*}{ Brain Region } & \multicolumn{2}{|c|}{ FR-HS } & \multicolumn{2}{|c|}{ MR-HS } & \multicolumn{2}{|c|}{ FL-HS } & \multicolumn{2}{|c|}{ ML-HS } \\
\hline & $\mathrm{FA}_{\mathrm{p}} / \mathrm{FA}_{\mathrm{c}}$ & $M D_{p} / M D_{c}$ & $F A_{p} / F A_{c}$ & $M D_{p} / M D_{c}$ & $F A_{p} / F A_{c}$ & $M D_{p} / M D_{c}$ & $F A_{p} / F A_{c}$ & $M D_{p} / M D_{c}$ \\
\hline $\mathrm{R}$ cingulum & $0.23 / 0.28$ & $0.85 / 0.82$ & $0.21 / 0.29$ & - & $0.31 / 0.35$ & - & - & $1.00 / 0.89$ \\
\hline $\mathrm{L}$ cingulum & - & $0.84 / 0.73$ & - & - & $0.24 / 0.34$ & - & $0.30 / 0.35$ & $1.00 / 0.73$ \\
\hline R ATWM;ILF & $0.23 / 0.40$ & $0.88 / 0.79$ & $0.25 / 0.34$ & $0.87 / 0.79$ & $0.32 / 0.37$ & $0.83 / 0.72$ & $0.19 / 0.24$ & - \\
\hline L ATWM;ILF & $0.34 / 0.40$ & $0.86 / 0.78$ & - & - & $0.31 / 0.36$ & $0.88 / 0.74$ & $0.22 / 0.28$ & $0.91 / 0.82$ \\
\hline R fornix & $0.29 / 0.33$ & $1.45 / 1.33$ & - & $1.69 / 1.48$ & $0.34 / 0.39$ & $1.70 / 1.32$ & $0.28 / 0.44$ & $1.87 / 1.57$ \\
\hline L fornix & - & - & $0.31 / 0.38$ & - & $0.32 / 0.41$ & $1.84 / 1.39$ & $0.34 / 0.44$ & $1.90 / 1.79$ \\
\hline R uncinate fasciculus & $0.30 / 0.43$ & $0.84 / 0.79$ & $0.36 / 0.45$ & $0.84 / 0.77$ & - & - & - & - \\
\hline $\mathrm{L}$ uncinate fasciculus & - & - & - & - & $0.47 / 0.51$ & $0.80 / 0.75$ & $0.32 / 0.39$ & $0.87 / 0.79$ \\
\hline CC genu & $0.36 / 0.59$ & $0.76 / 0.68$ & $0.64 / 0.78$ & $0.88 / 0.70$ & $0.38 / 0.49$ & $0.78 / 0.74$ & $0.40 / 0.56$ & $1.07 / 0.77$ \\
\hline CC body & $0.44 / 0.52$ & $1.12 / 0.92$ & $0.38 / 0.51$ & $1.69 / 1.29$ & - & - & $0.46 / 0.68$ & $1.09 / 0.81$ \\
\hline R ALIC & $0.49 / 0.55$ & $0.79 / 0.71$ & $0.26 / 0.29$ & $0.74 / 0.68$ & $0.65 / 0.76$ & $0.72 / 0.66$ & $0.54 / 0.65$ & $0.71 / 0.67$ \\
\hline R PLIC & $0.68 / 0.72$ & $0.71 / 0.62$ & $0.66 / 0.72$ & - & $0.59 / 0.66$ & $0.73 / 0.68$ & - & - \\
\hline L ALIC & - & - & - & - & $0.57 / 0.66$ & $0.75 / 0.65$ & $0.51 / 0.59$ & $1.00 / 0.68$ \\
\hline L PLIC & - & - & $0.65 / 0.69$ & - & $0.60 / 0.69$ & $0.73 / 0.66$ & - & - \\
\hline R external capsule & $0.34 / 0.43$ & $0.74 / 0.63$ & $0.39 / 0.46$ & - & - & - & $0.30 / 0.38$ & - \\
\hline L external capsule & $0.41 / 0.46$ & $0.78 / 0.74$ & $0.43 / 0.47$ & - & $0.39 / 0.45$ & $0.79 / 0.71$ & $0.34 / 0.42$ & $0.73 / 0.66$ \\
\hline $\mathrm{R}$ thalamus & $0.32 / 0.36$ & $0.86 / 0.75$ & $0.36 / 0.40$ & $0.81 / 0.66$ & $0.36 / 0.43$ & $0.70 / 0.64$ & $0.44 / 0.51$ & $0.79 / 0.75$ \\
\hline $\mathrm{L}$ thalamus & $0.31 / 0.35$ & $0.75 / 0.70$ & $0.29 / 0.34$ & $0.71 / 0.69$ & $0.31 / 0.37$ & $0.88 / 0.71$ & $0.30 / 0.36$ & $1.00 / 0.73$ \\
\hline R IFOF & $0.41 / 0.50$ & - & $0.29 / 0.37$ & $0.79 / 0.72$ & - & - & $0.30 / 0.46$ & - \\
\hline L IFOF & - & - & $0.30 / 0.35$ & - & $0.38 / 0.43$ & $0.83 / 0.75$ & $0.46 / 0.56$ & $0.80 / 0.75$ \\
\hline $\mathrm{R}$ acoustic radiation & $0.45 / 0.49$ & $0.79 / 0.69$ & - & - & - & - & - & - \\
\hline
\end{tabular}

Note:-The mean FA and MD $\left(x 10^{-3} \mathrm{~mm}^{2} / \mathrm{s}\right)$ values of significant clusters in patients along with those of control participants and corresponding WM regions are derived according to the Johns Hopkins University WM tractography atlas and the International Consortium for Brain Mapping DTI-81 WM atlas.

ATWM indicates anterior temporal lobe white matter; ALIC, anterior limb of the internal capsule; PLIC, posterior limb of the internal capsule; CC, corpus callosum; FA $/$ FA, fractional anisotropy of the patients/fractional anisotropy of the controls; FL-HS, female patients with left HS; FR-HS, female patients with right HS; IFOF, inferior frontooccipital fasciculus; ILF, inferior longitudinal fasciculus; L, left; $M D_{\mathrm{p}} / \mathrm{MD}_{\mathrm{c}}$, mean diffusivity of the patients/mean diffusivity of the controls; ML-HS, male patients with left HS; MR-HS, male patients with right HS; R, right; TBSS, tract-based spatial statistics.

structures. The cingulum ipsilateral to HS showed diffusion abnormalities in all groups, and the contralateral cingulum was also affected in all groups except in men with right HS. Involvement of the bilateral anterior temporal lobe WM, uncinate fasciculi, and internal and external capsules was marked in women with right $\mathrm{HS}$, whereas low FA values in these same structures were also observed to a much lesser extent in other groups. No WM structure was observed to have increased FA or decreased MD.

WM structures with reduced FA and elevated MD, and extent of WM alteration were displayed as TBSS maps in the Figure.

\section{DISCUSSION}

TBSS that allow whole-brain voxelwise analysis of multisubject data are particularly useful to identify the location of diffusivity abnormalities in groups of patients with particular diseases. ${ }^{17} \mathrm{FA}$, a noninvasive marker of WM integrity, can decrease because of axonal degeneration, reduced packing attenuation, coherence, or demyelination. Increased diffusivity can result from increased extracellular space from reduced cellular attenuation, increased membrane permeability, or loss of myelin sheaths. ${ }^{7}$ Changes in diffusion measures were commonly observed in the cingulum, fornix, superior longitudinal and inferior longitudinal fasciculi, corpus callosum, uncinate fasciculus, and the external and internal capsules in patients with TLE with HS. ${ }^{9,18,19}$ These changes may represent an underlying predisposing abnormality in production of TLE, abnormal networks in seizure propagation, reorganization of WM networks or solely damage caused by the seizures themselves, and use of antiepileptic drugs.

Similar to previous studies, ${ }^{3,5,9,18}$ our analyses revealed FA reduction and MD elevation in the temporal and extratemporal WM, more prominent ipsilateral to the HS regardless of patient sex and lateralization of the HS. However, these studies compared DTI changes in patients with TLE with or without HS, ${ }^{3}$ searched for a correlation between DTI findings and surgical outcome, ${ }^{9}$ and considered the effect of the age of seizure onset and the duration of epilepsy but did not consider the sex of the patients. ${ }^{4,20}$ In our study, we took the lateralization of HS into account in addition to patient sex because of the potential effect of lateralization on WM changes. ${ }^{4,19}$ With similar demographics, clinical features, and radiologic laterality of HS in the patient groups, we believe that our study design minimizes intersubject variations and reflects the effect of sexual dimorphism on this particular type of focal epilepsy.

Men with left HS, followed by women with right HS, showed the most extensive decreases in FA across the groups. When FA and MD were regarded overall, women with right HS showed the most widespread temporal and extratemporal WM diffusion abnormalities. In right HS, although the ipsilateral cingulum, uncinate fasciculus, internal and external capsules, and right acoustic radiation were involved extensively in women, these structures showed only a few separate clusters of voxels with low FA, if present, in men. In our study, the severity or extent of diffusion changes could not be solely attributed to the lateralization of HS, as more prominent WM diffusion changes were associated with different-sided HS between men and women. The previous finding ${ }^{18}$ of limited WM involvement in right HS compared with left HS might have partly resulted from the outweighing proportion of men in the patient group. On the contrary, in left HS, men showed more extensive alterations of anisotropy compared with women. By the same data, right HS in women and left HS in men showed more extensive FA decline in the WM of the brain. The same trend was also observed for MD in women. There- 


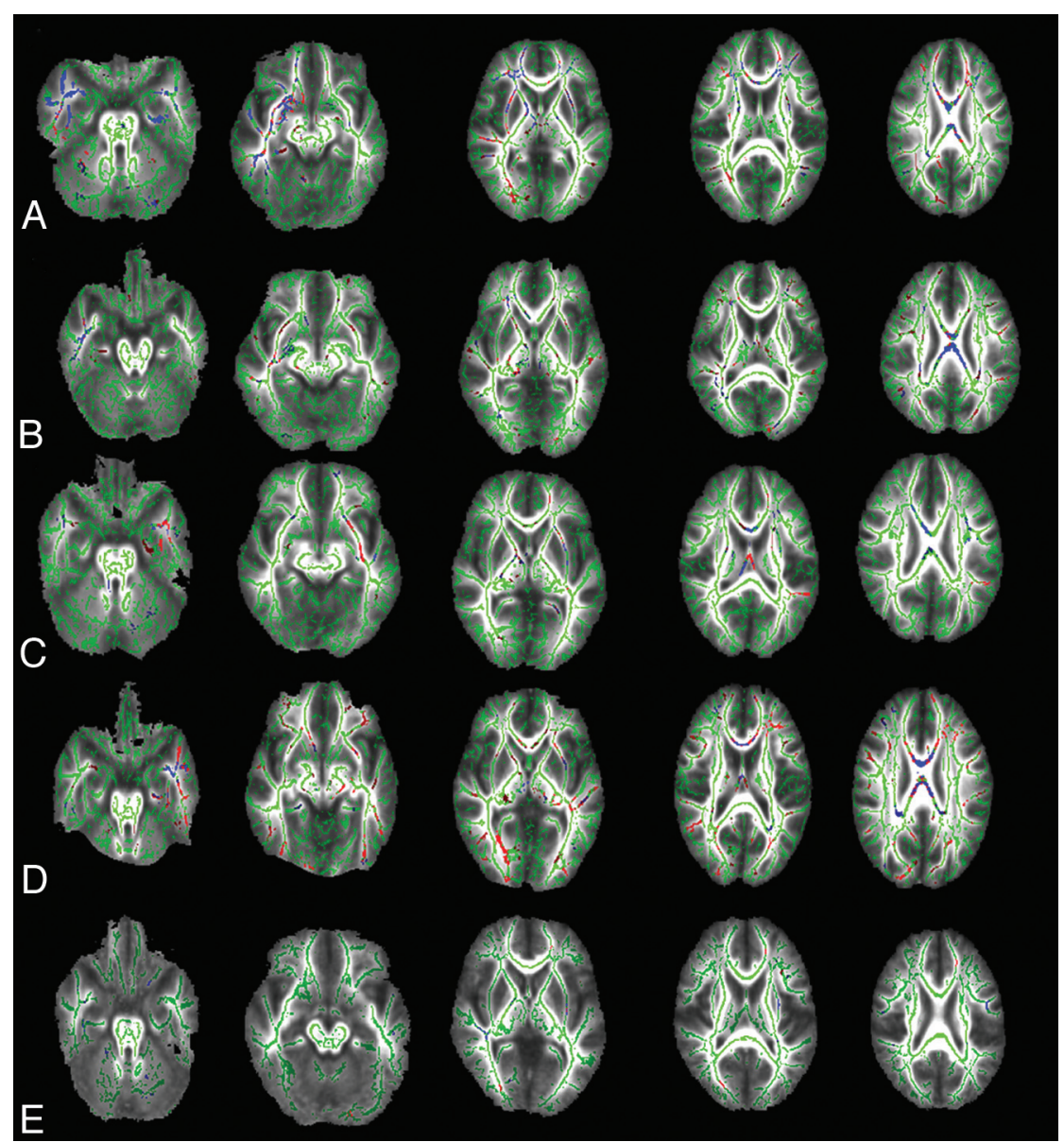

FIGURE. The TBSS (family-wise error-corrected threshold-cluster extend voxel $P$ maps) reveal regions of significantly reduced FA (red) and increased $\mathrm{MD}$ (blue) in female patients with right HS $(A)$, male patients with right HS (B), female patients with left HS (C), and male patients with left HS (D) compared with sex-matched and age-matched control groups at $P<.05$. E, The TBSS of women vs men within the whole control group show that men have few voxels with lower FA (red) and higher MD (blue) than women. FA skeleton that represents the center of the main WM tracts is shown in green on mean FA map.

fore, as hypothesized, the sex of the patient exerts an effect on the pattern of WM pathologic abnormalities, apart from the side of HS. Existing studies ${ }^{13,14}$ state that temporal lobe ictal behavior and affective symptoms are affected by patient sex rather than by dominant hemisphere and lateralization of the epileptic focus, and underlying different patterns of network abnormalities may be responsible for the variability in ictal symptoms.

Consistent with several studies ${ }^{21-23}$ on temporal lobe epilepsy with and without HS, the uncinate fasciculus connecting the frontal and temporal lobes showed the most extensive anisotropy and MD changes in women with right HS, along with changes in the anterior temporal lobe WM, internal and external capsules, and acoustic radiation. Tezer et $\mathrm{al}^{14}$ showed significantly increased ictal vocalization and speech in women with right HS. This finding may be explained by selective preferential disintegration of the uncinate fasciculus in a similar group of patients in our study. The uncinate fasciculus may consist of a pathway for propagation of synchronized neuronal firing from the mesial temporal lobe to the frontal and insulo-perisylvian areas. These areas frequently demonstrate hypometabolism on positron-emission tomography studies. Because of limited availability and high cost, positron-emission tomography scans were only performed in a few of our patients; therefore, these scans could not lead us to conclusions about seizure spread. We preferred to use continuous video-EEG records and interictal EEG records. In all patients with ipsilateral ictal abnormality on EEG, interictal EEG abnormality was observed bilaterally with predominance in the hemisphere ipsilateral to HS in 4 patients. However, routine EEGs and long-term EEG monitoring can be discordant, and in some patients, interictal epileptiform discharges can be seen only during long-term EEG monitoring. ${ }^{24}$

Among the 4 groups, men with left HS had the least amount of AI for both FA and MD. The presence of the most extensive FA, but not MD, alteration of WM in men with left HS fails to explain a probable relationship between $\mathrm{AI}$ and extension of pathologic abnormalities. Our samples of patients did not allow us to speculate about a relationship between $\mathrm{AI}$ and spread of epileptiform activity on EEGs either, because there were patients with contralateral EEG abnormalities in the groups of the patients with greater AI, but not in men with left HS with the least AI.

A limitation of our study was that many of these patients had 1 or more risk factors for HS including a history of febrile convulsions, meningitis, perinatal insult, and traumatic injury. Although MR imaging did not show a dual pathologic pattern in any of the patients, we cannot totally eliminate potential disrupting effects of these insults. With a limited number of patients, we were not able to separate FA effects of each risk factor. However, analyzing such effects of individual risk factors for HS could be a focus of future studies with more participants.

We did not measure $\mathrm{T} 2$ relaxation times of the hippocampi in the patients and control participants in this study. Rather, we rigorously included patients with an established diagnosis of unilateral HS on the basis of visual evaluation of epilepsy-dedicated MR imaging, electrophysiologic studies, and semiology.

DTI may improve our understanding of seizure propagation and relevant ictal behavior, electrophysiologic findings, and outcomes between male and female patients with HS. This development, in turn, may have a substantial effect in predicting surgical outcome and choosing appropriate treatment protocols in the future for such patients.

\section{CONCLUSIONS}

Our study demonstrated different patterns of HS-associated WM structural changes in men and women that may reflect previously 
reported sex differences in ictal behavior of the patients with HS. Patient sex should definitely be taken into account when the results of imaging studies on TLE with HS are being interpreted.

\section{REFERENCES}

1. Margerison JH, Corsellis JA. Epilepsy and the temporal lobes. A clinical, electroencephalographic, and neuropathological study of the brain in epilepsy, with particular reference to the temporal lobes. Brain 1966;89:499-30

2. Bronen RA, Cheung G, Charles JT, et al. Imaging findings in hippocampal sclerosis: correlation with pathology. AJNR Am J Neuroradiol 1991;12:933-40

3. Concha L, Beaulieu C, Collins DL, et al. White-matter diffusion abnormalities in temporal-lobe epilepsy with and without mesial temporal sclerosis. J Neurol Neurosurg Psychiatry 2009;80:312-19

4. Thivard L, Leheŕicy S, Krainik A, et al. Diffusion tensor imaging in medial temporal lobe epilepsy with hippocampal sclerosis. Neuroimage 2005;28:682-90

5. Gross DW. Diffusion tensor imaging in temporal lobe epilepsy. Epilepsia 2011;52:32-34

6. Cascino GD. A cerebral network reflecting reorganization in medial temporal lobe epilepsy. Epilepsy Curr 2006;6:46-48

7. Beaulieu $C$. The basis of anisotropic water diffusion in the nervous system - a technical review. NMR Biomed 2002;15:435-55

8. Otte WM, van Eijsden P, Sander JW, et al. A meta-analysis of white matter changes in temporal lobe epilepsy as studied with diffusion tensor imaging. Epilepsia 2012;53:659-67

9. Gross DW, Concha L, Beaulieu C. Extratemporal white matter abnormalities in mesial temporal lobe epilepsy demonstrated with diffusion tensor imaging. Epilepsia 2006;47:1360-63

10. Sowell ER, Peterson BS, Kan E, et al. Sex differences in cortical thickness mapped in $\mathbf{1 7 6}$ healthy individuals between 7 and 87 years of age. Cereb Cortex 2007;17:1550-60

11. Azari NP, Rapoport SI, Grady CL, et al. Gender differences in correlations of cerebral glucose metabolic rates in young normal adults. Brain Res 1992;574:198-208
12. Bava S, Boucquey V, Goldenberg D, et al. Sex differences in adolescent white matter architecture. Brain Res 2011;1375:41-48

13. Toth V, Fogarasi A, Karadi K, et al. Ictal affective symptoms in temporal lobe epilepsy are related to gender and age. Epilepsia 2010;51:1126-32

14. Tezer FI, Kurne A, Soylu AR, et al. Effects of lateralisation and gender on temporal lobe ictal behaviour associated with hippocampal sclerosis. Seizure 2004;13:418-24

15. Janszky J, Fogarasi A, Toth V, et al. Peri-ictal vegetative symptoms in temporal lobe epilepsy. Epilepsy Behav 2007;11:125-29

16. Janszky J, Ebner A, Szupera Z, et al. Orgasmic aura-a report of seven cases. Seizure 2004;13:441-44

17. Smith SM, Jenkinson M, Johansen-Berg H, et al. Tract-based spatial statistics: voxelwise analysis of multi-subject diffusion data. Neuroimage 2006;31:1487-505

18. Focke NK, Yogarajah M, Bonelli SB, et al. Voxel-based diffusion tensor imaging in patients with mesial temporal lobe epilepsy and hippocampal sclerosis. Neuroimage 2008;40:728-37

19. Ahmadi ME, Hagler DJ Jr, McDonald CR, et al. Side matters: diffusion tensor imaging tractography in left and right temporal lobe epilepsy. AJNR Am J Neuroradiol 2009;30:1740-47

20. Meng L, Xiang J, Kotecha R, et al. White matter abnormalities in children and adolescents with temporal lobe epilepsy. Magn Reson Imaging 2010;28:1290-98

21. Rodrigo S, Oppenheim C, Chassoux F, et al. Uncinate fasciculus fiber tracking in mesial temporal lobe epilepsy. Initial findings. Eur Radiol 2007;17:1663-68

22. Diehl B, Busch RM, Duncan JS, et al. Abnormalities in diffusion tensor imaging of the uncinate fasciculus relate to reduced memory in temporal lobe epilepsy. Epilepsia 2008;49:1409-18

23. Kim CH, Chung CK, Koo BB, et al. Changes in language pathways in patients with temporal lobe epilepsy: diffusion tensor imaging analysis of the uncinate and arcuate fasciculi. World Neurosurg 2011;75:509-16

24. Cascino GD, Trenerry MR, So EL, et al. Routine EEG and temporal lobe epilepsy: relation to long-term EEG monitoring, quantitative MRI, and operative outcome. Epilepsia 1996;37:651-56 